\title{
Carbon Monoxide Poisoning in the Veterans Health Administration, 2010 - 2016
}

\author{
Gina Oda*1, Russell Ryono', Cynthia A. Lucero-Obusan'1, Patricia Schirmer ${ }^{1}$ and \\ Mark Holodniy, 2
}

${ }^{1}$ Public Health Surveillance and Research, Department of Veterans Affairs, Palo Alto, CA, USA; 'Stanford University, Stanford, CA, USA

\section{Objective}

To describe characteristics of Veterans Health Administration (VHA) patients with ICD 9/10 CM inpatient discharge and/or emergency department (ED)/urgent care outpatient encounter codes for carbon monoxide $(\mathrm{CO})$ poisoning.

\section{Introduction}

It is estimated that in the United States (US), unintentional non-fire related $\mathrm{CO}$ poisoning causes an average of 439 deaths annually, and in 2007 confirmed CO poisoning cases resulted in 21,304 ED visits and 2,302 hospitalizations (71 per million and 8 per million population, respectively $)^{1}$. Despite the significant risk of morbidity and mortality associated with CO poisoning, existing surveillance systems in the United States are limited. This study is the first to focus specifically on $\mathrm{CO}$ poisoning trends within the VHA population.

\section{Methods}

Queries were performed in VA Praedico ${ }^{\mathrm{TM}}$ Public Health Surveillance System for inpatient discharges and emergency room and urgent care outpatient visits with ICD 9/10 CM codes for CO poisoning from $1 / 1 / 2010-6 / 30 / 2016$. A dataset of unique patient encounters with $\mathrm{CO}$ poisoning was compiled and further classified as accidental, self-harm or unspecified. Patients with carboxyhemoglobin ( $\mathrm{COHb}$ ) blood level measurements $\geq 10 \%^{2}$ for the same timeframe were extracted and merged with the $\mathrm{CO}$ poisoning dataset. We analyzed for demographic, geographic and seasonal variables. Rates were calculated using total unique users of VHA care for matching time frame and geographic area as denominators.

\section{Results}

There were a total of 671 unique VHA patients identified with CO poisoning. Of these, $298(44 \%)$ were classified as accidental, 104 (15\%) self-harm, and $269(40 \%)$ unspecified. A total of 6 patients died within 30 days of their coded diagnosis, however only 1 of these was directly attributable to $\mathrm{CO}$ poisoning. The overall rate of CO poisoning over the study time frame was 18 per million unique users of VHA care. $\mathrm{CO}$ poisoning diagnoses were obtained from $396(59 \%)$ outpatients, $216(32 \%)$ inpatients, and 59 (9\%) patients with both and outpatient visit and inpatient admission. Patients with self-harm classification were less likely to be seen in the ED (only $24(6 \%)$ unique patients compared to 190 (48\%) accidental and $182(46 \%)$ unspecified classifications). Of patients seen in the ED and subsequently admitted, patients with the classification of accidental poisoning made up the largest percentage with 36 unique patients (61\%). There were $71(11 \%)$ females compared to $600(89 \%)$ males. The highest represented age group was 45-64 with 342 unique patients $(51 \%)$. Rates by US Census Region were highest in the Midwest and Northeast (27 and 23 per million unique users, respectively) compared to the West and South (15 and 13 per million unique users, respectively) (Figure 1). Accidental CO poisonings showed a seasonal pattern with peaks occurring in late fall, winter, and early spring months (Figure 2). CO poisonings classified as unspecified had a similar but less pronounced pattern, while those classified as self-harm were too few to observe any pattern over time. $\mathrm{COHb}$ blood levels $\geq 10 \%$ were present in $111(17 \%)$ of patients with CO poisoning codes. Of patients with $\mathrm{COHb}$ measures $\geq 10 \%$, those with self-harm classification were least represented with only 7 unique patients $(6 \%)$. Accidental and unspecified classifications were equally represented with $53(48 \%)$ and $51(46 \%)$ unique patients, respectively.

\section{Conclusions}

The impact of CO poisoning on the VHA patient population has not been well studied. The geographic distribution of the majority of cases in the Midwest and Northeast, and the seasonal distribution of accidental cases in colder months seems to be appropriate with respect to what is known of unintentional $\mathrm{CO}$ poisoning as often associated with heat-generating sources ${ }^{3}$. Opportunities for further investigation include how potential $\mathrm{CO}$ poisoning cases are evaluated in VHA given the low percentage of cases with $\mathrm{COHb}$ blood level measurements.

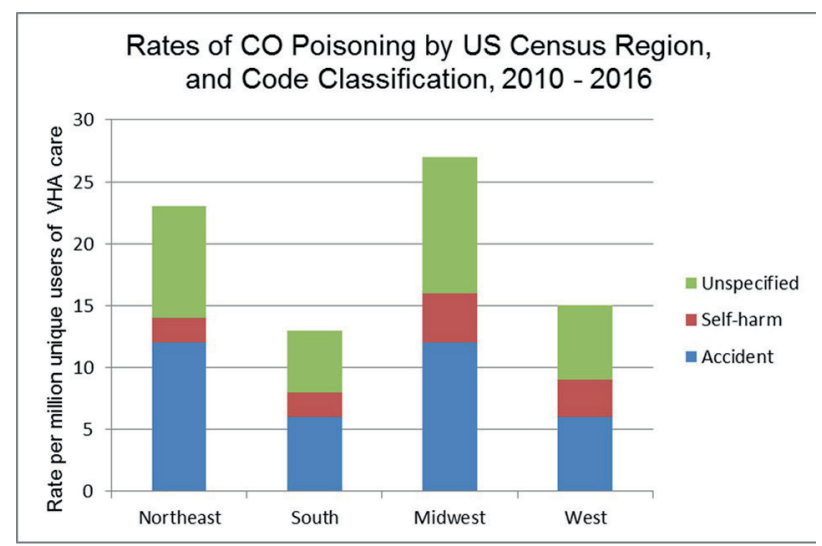

Figure 1 
ISDS 2016 Conference Abstracts

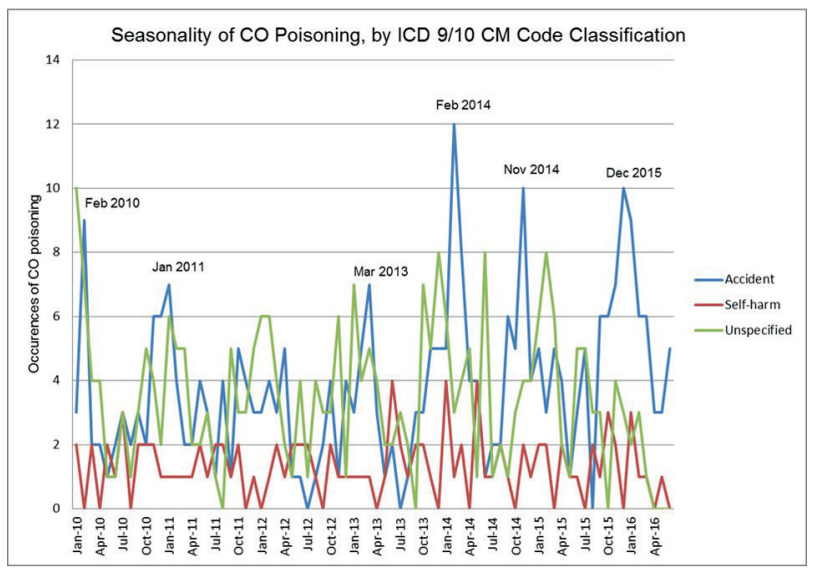

Figure 2

\section{Keywords}

carbon monoxide; Veterans Health Administration; non-infectious disease surveillance

\section{References}

1. Iqbal S, Clower JH, King M, Bell J, Yip F. National Carbon Monoxide Poisoning Surveillance Framework and Recent Estimates. Public Health Rep. 2012 Sep-Oct;127:486-496.

2. Council of State and Territorial Epidemiologists. Public Health Reporting and National Notification for Carbon Monoxide Poisoning, Position Statement 13-EH-01 Available from: URL: http://c.ymcdn. com/sites/www.cste.org/resource/resmgr/PS/13-EH-01.pdf

3. Centers for Disease Control and Prevention. Unintentional non-fire related carbon monoxide-exposures- United States, 2001-2003. MMWR 2005;54(02);36-39.

\section{*Gina Oda}

E-mail: gina.oda@va.gov 